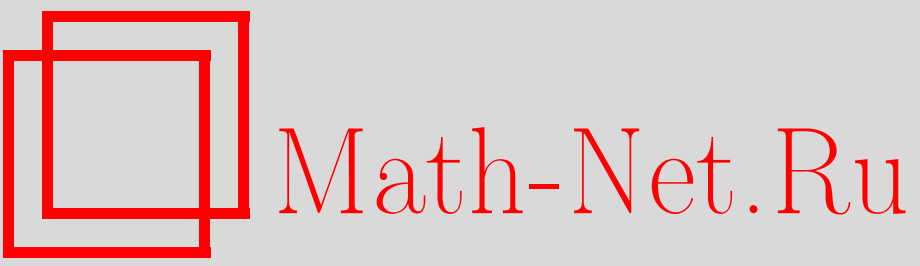

А. Е. Куршев, Моментная оценка для процесса Леви, УМН, 2002, том 57, выпуск $3,157-158$

DOI: https://doi.org/10.4213/rm522

Использование Общероссийского математического портала Math-Net.Ru подразумевает, что вы прочитали и согласны с пользовательским соглашением

http://www.mathnet.ru/rus/agreement

Параметры загрузки:

IP : 54.157 .27 .8

26 апреля 2023 г., $17: 11: 56$ 


\section{МОМЕНТНАЯ ОЦЕНКА ДЛЯ ПРОЦЕССА ЛЕВИ}

\section{A. Е. КУРШЕВ}

Пусть $X$ - безгранично делимая случайная величина. Предположим, что $\mathrm{E}|X|<\infty, \mathrm{E} X=0$ и $X$ не содержит гауссовской компоненты. Как известно, характеристическая функция $X$ может быть представлена в виде

$$
\mathrm{E} e^{i \theta X}=\exp \left\{\int_{\mathbb{R}}\left(e^{i \theta x}-1-i \theta x\right) \nu(d x)\right\}
$$

где $\nu-\sigma$-конечная борелевская мера с $\nu(\{0\})=0$ и

$$
\int_{\mathbb{R}}\left(|x| \wedge\left|x^{2}\right|\right) \nu(d x)<\infty
$$

В случае, когда $\nu(\mathbb{R})>0$, определим $l_{1}=l_{1}(\nu)>0$ как решение уравнени

$$
\int_{\mathbb{R}}\left(\frac{|x|}{l_{1}} \wedge \frac{\left|x^{2}\right|}{l_{1}^{2}}\right) \nu(d x)=1 .
$$

В силу (1) $l_{1}$ определяется однозначно.

В [1] предлагаются оценки для $\mathrm{E}|X|$ как функции от $l_{1}(\nu)$, а именно, утверждается

TEOPEMA 1.

$$
\frac{1}{4} l_{1}(\nu) \leqslant \mathrm{E}|X| \leqslant \frac{17}{8} l_{1}(\nu) .
$$

В настоящей работе дано обобщение теоремы 1 на случай $L^{n}$-нормы процессов Леви.

Пусть $X=\left(X_{t}\right)_{t \geqslant 0}$ - процесс Леви с $\mathrm{E}\left|X_{t}\right|^{n}<\infty, \mathrm{E} X_{t}=0$ и триплетом $(0,0, \nu)$. Как известно,

$$
\mathrm{E} e^{i \theta X_{t}}=\exp \left\{\int_{\mathbb{R}}\left(e^{i \theta x}-1-i \theta x\right) \nu_{t}(d x)\right\}
$$

где

$$
\nu_{t}(d x)=t \cdot \nu(d x)
$$

Если $\nu(\mathbb{R})>0$, зададим $l_{n}=l_{n}(\nu)>0$ с помощью равенства

$$
\int_{\mathbb{R}}\left(\frac{|x|^{n}}{l_{n}^{n}} \wedge \frac{|x|^{n+1}}{l_{n}^{n+1}}\right) \nu(d x)=1 .
$$

Поскольку $\mathrm{E}\left|X_{t}\right|^{n}<\infty, l_{n}$ определяется однозначно.

Рассмотрим разложение $X_{t}=Y_{t}+Z_{t}$, где $Y_{t}$ и $Z_{t}$ независимы, с $\mathrm{E} Y_{t}=\mathrm{E} Z_{t}=0$ и характеристическими функциями

$$
\begin{aligned}
& \mathrm{E} e^{i \theta Y_{t}}=\exp \left\{t \int_{|x|<l_{n}}\left(e^{i \theta x}-1-i \theta x\right) \nu(d x)\right\} \\
& \mathrm{E} e^{i \theta Z_{t}}=\exp \left\{t \int_{|x| \geqslant l_{n}}\left(e^{i \theta x}-1-i \theta x\right) \nu(d x)\right\} .
\end{aligned}
$$


Лемма 1.

$$
\begin{array}{ll}
\mathrm{E}\left|Y_{t}\right|^{m} \leqslant a_{m} \int_{|x|<l_{n}}|x|^{m} \nu(d x), & m=2, \ldots, n+1, \\
\mathrm{E}\left|Z_{t}\right|^{m} \leqslant b_{m} \int_{|x| \geqslant l_{n}}|x|^{m} \nu(d x), & m=1, \ldots, n,
\end{array}
$$

әде

$$
\begin{aligned}
& a_{m}=\frac{1}{\lambda} \sum_{\substack{k_{2}, \ldots, k_{m} \geqslant 0 \\
\sum_{j=2}^{m} j k_{j}=m}} \frac{m !}{k_{2} ! \cdots k_{m} !}\left(\frac{\lambda t}{2 !}\right)^{k_{2}} \cdots\left(\frac{\lambda t}{m !}\right)^{k_{m}} \\
& b_{m}=\frac{1}{\mu} \sum_{r=0}^{m} \sum_{\substack{k_{1}, \ldots, k_{r} \geqslant 0 \\
\sum_{j=1}^{r} j k_{j}=r}} \frac{m !}{k_{1} ! \cdots k_{r} !(m-r) !}\left(\frac{\mu t}{1 !}\right)^{k_{1}} \cdots\left(\frac{\mu t}{r !}\right)^{k_{r}}(\mu t)^{m-r}, \\
& \lambda=\nu\left(\left\{|x|<l_{n}\right\}\right), \quad \mu=\nu\left(\left\{|x| \geqslant \ln _{n}\right\}\right) .
\end{aligned}
$$

Неравенство (4) получается с помощью $m$-кратного дифференцирования характеристической функции $Y_{t}$ по $\theta$. Соотношение (5) доказывается для составного процесса Пуассона, совпадающего по распределению с $Z_{t}$.

ТеОрема 2. Справедливы неравенства

$$
c_{n}(t) l_{1}^{n} \leqslant \mathrm{E}\left|X_{t}\right|^{n} \leqslant C_{n}(t) l_{n}^{n},
$$

əде

$$
\begin{gathered}
c_{n}(t)= \begin{cases}\left(\frac{t}{4}\right)^{n}, & t \leqslant 2, \\
\left(\frac{t}{(4+6 t)^{1 / 2}}\right)^{n}, & t>2,\end{cases} \\
C_{n}(\lambda, \mu, t)=\max _{u \in[0,1]}\left(\left(a_{n+1} u\right)^{1 /(n+1)}+\left(b_{n}(1-u)\right)^{1 / n}\right)^{n} .
\end{gathered}
$$

Нижняя оценка получается для $n=1$ из доказательства нижней оценки в (2) с использованием (3) и обобщается для произвольного момента с помощью неравенства Ляпунова. Технической основой доказательства верхней оценки является лемма 1.

СлЕдСТвИЕ.

$$
c_{1}(t) l_{1} \leqslant \mathrm{E}\left|X_{t}\right| \leqslant\left(2 t+\frac{1}{8}\right) l_{1}
$$

ЗАмЕчАниЕ. В случае $t=1$, как и следовало ожидать, (6) представляет собой утверждение теоремы 1.

\section{СПИСОК ЛИТЕРАТУРЫ}

[1] M. B. Marcus, J. Rosinski // Electron. Comm. Probab. 2001. V. 6. P. 15-29.

Московский государственный университет им. М. В. Ломоносова 